\title{
Emergence of Novel Agents for Treatment of Hodgkin Lymphoma
}

\author{
Nahla AM Hamed* \\ Department of Hematology, Alexandria University, Egypt
}

Submission: December 02, 2017; Published: December 14, 2017

"Correspondence Address: Nahla AM Hamed, Professor of Hematology, Faculty of Medicine, Alexandria University, Egypt, Email: drhamedn@hotmail.com

\begin{abstract}
cHL patients are generally classified in 1 of 3 groups: early-stage favorable, early-stage unfavorable or advanced-stage disease. Prognostication in advanced-stage patients is defined by the IPS. Patients with low-risk IPS are treated with 6 cycles of standard ABVD, whereas high-risk patients are initially treated with the more intensive German-derived regimen, escalated BEACOPP. Treatment may be further refined through PETadapted therapy. The optimal chemotherapy for advanced stage HL can be endlessly debated. Based on recent study results, two classes of drugs stand out as highly active in advanced HL: antibody-drug conjugates (BV) and programmed death 1 inhibitors (Nivolumab). There are abundant salvage therapy options for patients with R/R HL. The choice of salvage chemotherapy therapy is becoming increasingly difficult in the era of novel agents.
\end{abstract}

Abbreviations: cHL: Classical Hodgkin lymphoma; IPS: International Prognostic Score; EBV: Epstein Barr Virus; H/RS: Hodgkin and Reed Sternberg; ASCT: autologous stem cell transplantation; BV: Brentuximab-Vedotin; PD-1: Programmed Death 1; MMAE: Monomethyl Auristatin E; CAR-T: Chimeric Antigen Receptor-modified T cell; GVHD: Graft-Versus-Host Disease; HL: Hodgkin lymphoma; PD-L1: Programmed Death Receptor Ligand 1; PD-L2: Programmed Death Receptor Ligand 2; R/R: Relapsed/Refractory; TARC: Thymus And Activation-Regulated Chemokine; TME: Tumor Microenvironment

\section{Introduction}

cHL accounts for about $10 \%$ of all lymphomas, with approximately 9000 new cases in the United States in 2013 and 1200 deaths [1]. cHL is derived from germinal center B cells [2]. Its pathogenesis remains poorly understood [3]. Molecular alterations involved in cHD development are only partially known [4] due to rarity of tumor cells (HRS- cells) in the lymph node [2]. Tumor immune evasion through PD-1 augmentation appears to play a major role in the oncogenesis of cHL [5].

\section{Hodgkin and Reed Sternberg (HRS) cells}

$\mathrm{H} / \mathrm{RS}$ cells are large CD30+ tumor-infiltrating cells that are derived from germinal center B cells and lack immunoglobulin expression [3]. They usually constitute $<5 \%$ of an extensive ineffective inflammatory/ immune micro-environment [5]. Despite this extensive polymorphous inflammatory infiltrate, there is poor antitumor immune response to neoplastic HRS cells [5]. H/RS cells are highly interactive with this microenvironment through direct cell contacts and production of various cytokines and chemokines [3]. HRS cells demonstrate high expression of the immunoregulatory glycan-binding protein and galectin-1 resulting in a type 2 T-helper cell and T-regulatory cell skewed tumor micro-environment [5]. H/RS cells may escape apoptosis within germinal center due to alteration of apoptosis regulators expression [3]. Genetic alterations in NFKB pathway and the imbalance of T regulatory and TH17 lymphocytes has been recognized as critical pathogenetic mechanism involved in immune escape and blockade of apoptosis in Reed-Sternberg cells [4].

\section{Advanced-stage disease}

Advanced-stage disease refers to patients with Ann Arbor stage III/IV disease and patients with high-risk stage II disease. Prognostication in advanced-stage patients is defined by the IPS. Patients with an IPS greater than or equal to 3 have inferior treatment outcomes and identified as potentially requiring more intensive therapy [6]. cHL is a highly curable lymphoma and about $80 \%$ of patients can be cured with modern treatment strategies [3]. Refractory cHL represent 5 to $10 \%$ of cHL [3] and are more frequent in patients with advanced HL [1]. Many of these patients have a poor overall survival of $26 \%$ at 5 years [3]. A better biological characterization of primary refractory patients might allow the use of targeted therapeutic strategies earlier during the course of the disease [3]. Relapse after first-line treatment occurs in $25-30 \%$ of cHD patients [7]. 
Secondary therapy only cures approximately $50 \%$ of patients with no reliable biomarkers to identify the patients in which the salvage treatment regimen fails [7]. The current standard care for $\mathrm{R} / \mathrm{R}$ cHD is salvage chemotherapy followed by high dose chemotherapy and ASCT [1]. At the present time, there is a range of chemotherapy-based and novel-agent-based salvage options that can lead to excellent overall response rate and complete response rates [1].

\section{Suggested predictors of response to therapy}

Understanding cHL associated immunosuppression and the immune reconstitution after treatment may be the key to develop new prognostic factors and treatment strategies [8]. A correlation between markers of cell activation and/ or differentiation, cell cycle and apoptosis deregulation, EBV detection in the neoplastic $\mathrm{H} / \mathrm{RS}$ cells and the clinical outcome of cHL patients have been reported [3]. CD20 expression in H/RS cells is significantly higher in responders compared to refractory cHL. CD20 resembles a Ca2+ ion channel and is involved in signal transduction for B-cell differentiation and proliferation. An increase of $\mathrm{Ca} 2+$ permeability in $\mathrm{H} / \mathrm{RS}$ cells along with chemotherapy and/or radiotherapy could decrease apoptotic resistance or even activate programmed cell death [3].

a. Serum levels of soluble CD30 and some interleukins might provide additional prognostic information to the clinical models [3].

b. Chan et al. [7] develop a novel model based on the TME gene expression profile of relapsed patients that have superior predictive properties for response to ASCT.

c. The measurement of the serum chemokine TARC could help identifying patients who may require new drugs like $\mathrm{BV}$ or nivolumab or pembrolizumab before ASCT, in order to achieve a durable remission [9].

\section{Novel agents}

A large number of novel agents have been approved and being investigated for HL treatment. Many of these agents have shown durable responses with improved safety profiles [10]. Based on recent study results, two classes of drugs stand out as highly active in advanced HL: antibody-drug conjugates (BV) and PD-1 inhibitors (Nivolumab) [11].

\section{Brentuximab Vedotin (Anti-CD30)}

$\mathrm{BV}$ is an antibody-drug conjugates that selectively target CD30 on the surface of the malignant HL cells to achieve its therapeutic effect [10] with minimal collateral damage to normal tissue [11]. In cHL, CD30 expression is highly expressed on ReedSternberg cells with normal limited expression on activated $\mathrm{B}$ and $\mathrm{T}$ cells only [10]. BV consists of a chimeric monoclonal antibody against human CD30 coupled to the antimicrotubule agent MMAE using a peptide linker. BV binds CD30 on the surface of the malignant HL cells and after being internalized, it releases MMAE which prevents tubulin polymerization, a protein essential for cell division, causing cell cycle arrest and apoptosis [11]. BV was approved by the FDA for treatment of HL patients who has failed either ASCT or two other chemotherapy regimens and are not eligible for transplant [10]. The incorporation of BV either as a single agent or as part of salvage therapy for R/R HL patients regimen is appealing.

Two strategies have evolved to study BV as part of salvage therapy for R/R HL patients: sequential administration of BV with chemotherapy and combined chemo-immunotherapy [12]. $\mathrm{BV}$ can be used as a salvage option in prior BV responders [1]. As a single agent, BV is used at a dose of $1.8 \mathrm{mg} / \mathrm{kg}$ (up to $180 \mathrm{mg}$ ) intravenously every 3 weeks up to 16 cycles, disease progression, or unacceptable toxicity. BV is generally well tolerated [11] and has improved the outcomes of cHL patients [13]. The PETnegative rate after 2 to 4 doses of single agent $\mathrm{BV}$ is $30 \%$ to $40 \%$ in heavily pretreated patients. However, many eventually become refractory to BV. Duration and depth of response are important, as patients are often young and otherwise healthy [13]. BV is under study as part of front-line therapy [1].

It may be difficult to interpret data on BV-based secondline regimens in which patients were naïve to $\mathrm{BV}$. Introduction of BV into second-line therapy allow its use as a bridge to ASCT with fewer side effects than conventional chemotherapy [1]. Post-ASCT consolidation with BV should be reserved for patients with multiple risk factors because the adverse effects are significant (namely, neuropathy) [12]. Cumulative peripheral neuropathy is the leading cause of drug discontinuation in some clinical trials. It may be severe (up to $31 \%$ at least grade 2) [11]. Sensory neuropathy is more commonly seen (42\%) than motor neuropathy (11\%). Median time to onset of neuropathy was about 12 weeks. Some improvement of symptoms (of at least one grade) was experienced in $80 \%$ of patients with drug discontinuation but complete resolution was observed in only half of the cases [11]. Other uncommon serious adverse events are progressive multifocal leukoencephalopathy and pancreatitis. The incidence of febrile neutropenia was extremely low [11].

\section{Nivolumab (humanized immunoglobulin gamma-4 kappa anti-PD-1 receptor monoclonal antibody)}

Under physiological conditions, PD-1 pathway activation via PD-L1 and PD-L2 engagement limits T-cell-mediated immune responses [13]. In cHL, recurrent amplification of 9q24.1 locus increase the abundance of the PD-1 ligands, PD-L1 and PD-L2, on the tumor cell surface [14] and promote their induction through increase JAK-STAT signaling. JAK2 locus is also located on chromosome 9p24.1 [13]. In addition, latent EBV infection may contribute to increase PD-L1 and PD-L2 expression in EBVpositive HL (around $40 \%$ of cHL cases) [5]. The increased PDL1 and PD-L2 expression by Reed-Sternberg cells may enable them to evade immune surveillance [13]. Disrupting the PD1:PD-L1/2 axis by nivolumab overcome the immune tolerance induced by cancer cells, thereby releasing the immune system 
for an effective anti-cancer response [11]. Nivolumab received accelerated FDA approval in May 2016 for treatment of R/R cHL progressing after ASCT and BV treatment [15].

Nivolumab produces durable responses, even in heavily pretreated patients with standard cytotoxic agents [13]. Nivolumab was associated with an overall response rate of $87 \%$ and a rate of progression-free survival of $86 \%$ at 24 weeks [16]. The most common adverse effects of this class are dermatologic (rash and pruritus), metabolic (lipid changes, hyperglycemia, hypoalbuminemia, and electrolyte imbalances), hematologic (anemia and lymphopenia), gastrointestinal (changes in bowel habits and nausea/vomiting), respiratory (cough and dyspnea), fatigue, abnormal liver enzymes, and arthralgia [10]. Adverse events were mainly of grade 1 or 2 [16]. Immune related adverse effects are the result of the immune-stimulatory effects of these drugs. They are uncommon but have pleomorphic presentations.

Potentially affected organs are the lungs (pneumonitis), the endocrine system (hypophysitis, thyroiditis, and adrenal insufficiency), the skin (toxic epidermal necrolysis), and the gastrointestinal tract (colitis and pancreatitis). These events can progress rapidly to cause significant morbidity or even death. Treatment must be individualized, hold therapy (for mild cases) and systemic steroids (for moderate to severe cases). Invasive testing (i.e. bronchoscopies and colonoscopies) are often needed to rule out infection or tumor progression. Caution is advised with utilization of this class in patients with a documented history of poorly controlled autoimmune disorders or for patients who have failed allogeneic SCT and have active GVHD [10].

\section{Future therapy}

The identification of new biomarkers in HL may provide potential targets for the development of new treatment agents. HRS cells display simultaneous activation of different cellsurvival pathways, including NF- $\mathrm{B}$, JAK/STAT, PIP3K/AKT/ mTOR, NOTCH and RAF/MEK/ERK [17]. In recent years, new agents have been developed and tested in HL with encouraging results [11].

\section{CAR-T therapy}

This involves genetically re-engineering the patient's effector immune cells (T cells) to recognize and eliminate tumor cells via modifications to the $\mathrm{T}$ cell receptor. Preclinical models demonstrate encouraging activity if the same principle has been applied to target CD30 -expressed on the surface of HL cells [11].

\section{Small molecule inhibitors of cell signaling (PI3K $\delta$ inhibitor and JAK2 kinase inhibitor)}

The JAK-STAT and PI3K pathways play an important role in the survival of RS cells and are deregulated in cHL. The combination of agents targeting these pathways may have a synergistic effect [10]. a. A phase 1 dose escalation trial of combination of INCB040093, a PI3K $\delta$ inhibitor and INCB039110, a selective JAK1 inhibitor, in R/R cHL patients showed an acceptable safety profile. The ORR was 50\% [10].

b. A phase 2 trial investigates the efficacy of pembrolizumab (PD-1 inhibitor) after ASCT in R/R cHL (NCT02362997) [10]. Eligible patients in this study were those who failed BV treatment [12].

c. Clinical trials combining PD-1 inhibitors to Bruton tyrosine kinase inhibitors (NCT02362035), bispecific NKcell engager antibodies (NCT02665650), BV (NCT01896999 and NCT02572167), other immune checkpoint inhibitors (NCT01896999, NCT02304458, and NCT01592370) or standard cytotoxic chemotherapy (NCT02181738) are under active development in an attempt to increase efficacy [10].

\section{Conclusion}

There have been a large number of novel agents approved and currently being investigated for treatment of HL. Many of these agents have shown durable responses with improved safety profiles. This may modify the treatment paradigm in the future.

\section{References}

1. Nikolaenko L, Chen R, Herrera AF (2017) Current strategies for salvage treatment for relapsed classical Hodgkin lymphoma. Ther Adv Hematol 8(10): 293-302.

2. Tiacci E, Penson A, Schiavoni G, Ladewig E, Fortini E, et al. (2016) New recurrently mutated genes in classical Hodgkin lymphoma revealed by whole-exome sequencing of microdissected tumor cells. Blood 128: 1088.

3. Canioni D, Deau-Fischer B, Taupin P, Ribrag V, Delarue R, et al. (2009) Prognostic significance of new immunohistochemical markers in refractory classical Hodgkin lymphoma: A study of 59 cases. PLoS ONE 4(7): e6341.

4. Silva PB, Real JM, Baiocchi OCG, Esteves GH, Junior JG, et al. (2016) Gene networks and conical pathways in classical Hodgkin lymphoma patients. Blood 128: 5298.

5. Gay ND, Okada CY, Chen AI, Scott EC (2017) Targeting the programmed cell death 1 pathway in Hodgkin lymphoma: the place of nivolumab. Ther Adv Hematol 8(5): 175-180.

6. Allen PB, Gordon L (2017) Frontline therapy for classical Hodgkin lymphoma by stage and prognostic factors. Clin Med Insights Oncol 11: 1-10.

7. Chan FC, Mottok A, Gerrie AS, Power MM, Savage KJ, et al. (2016) A novel prognostic model based on tumor microenvironment biology in relapse biopsies predicts post-autologous stem cell transplantation outcomes in classical Hodgkin lymphoma. Blood 128: 1093.

8. Saliva PB, Real JM, Ferreira LRP, Esteves GH, Junior JG, Penna AMD, et al. (2016) Impact of treatment on IL-10, CTLA-4 and PD-L1 gene 


\section{Cancer Therapy \& Oncology International Journal}

expression in patients with classical Hodgkin lymphoma. Blood 128 5290.

9. Viviani S, Spina F, Mazzocchi A, Galbiati M, Crippa F, et al. (2016) TARC before ASCT is highly predictive of outcome in patients with relapsed or refractory classical Hodgkin lymphoma after first line therapy. Blood 128: 3009.

10. Grover NS, Park SI (2015) Novel targeted agents in Hodgkin and NonHodgkin lymphoma therapy. Pharmaceuticals (Basel) 8(3): 607-636.

11. Villasboas JC, Ansell SM (2016) Recent advances in the management of Hodgkin lymphoma F1000Res.

12. Moskowitz C (2016) Novel agents and strategies in transplant-eligible patients with relapsed and refractory Hodgkin lymphoma. Hematology Am Soc Hematol Educ Program 2016(1): 331-338.

13. Younes A, Santoro A, Shipp M, Zinzani PL, Timmerman JM, et al. (2016) Nivolumab for classical Hodgkin lymphoma after autologous stem-cell transplantation and brentuximab vedotin failure: a prospective phase 2 multi-cohort study. Lancet Oncol 17(9): 1283-1294.

14. Falchi L, Sawas A, Deng C, Amengual JE, Colbourn DS, et al. (2016) High rate of complete responses to immune checkpoint inhibitors in patients with relapsed or refractory Hodgkin lymphoma previously exposed to epigenetic therapy. J Hematol Oncol 9(1): 132-153.

15. Sandoval-Sus JD, Mogollon-Duffo F, Patel A, Visweshwar N, Laber DA, et al. (2017) Nivolumab as salvage treatment in a patient with HIVrelated relapsed/refractory Hodgkin lymphoma and liver failure with encephalopathy. J Immunother Cancer 5: 49.

16. Ansell SM, Lesokhin AM, Borrello I, Halwani A, Scott EC, et al. (2015) PD-1 blockade with nivolumab in relapsed or refractory Hodgkin's lymphoma. N Engl J Med 372(4): 311-319.

17. Derenzini E, Younes A (2011) Predicting treatment outcome in classical Hodgkin lymphoma: genomic advances. Genome Medicine 3(4): 26.

Your next submission with Juniper Publishers will reach you the below assets

- Quality Editorial service

- Swift Peer Review

- Reprints availability

- E-prints Service

- Manuscript Podcast for convenient understanding

- Global attainment for your research

- Manuscript accessibility in different formats

( Pdf, E-pub, Full Text, Audio)

- Unceasing customer service

Track the below URL for one-step submission https://juniperpublishers.com/online-submission.php 